\title{
Experimental study of the sub-wavelength imaging by a wire medium slab
}

\author{
Pavel A. Belov, Yan Zhao, Sunil Sudhakaran, Akram Alomainy, and Yang Hao \\ Department of Electronic Engineering, Queen Mary University of London, \\ Mile End Road, London, E1 4NS, United Kingdom
}

\begin{abstract}
An experimental investigation of sub-wavelength imaging by a wire medium slab is performed. A complex-shaped near field source is used in order to test imaging performance of the device. It is demonstrated that the ultimate bandwidth of operation of the constructed imaging device is $4.5 \%$ that coincides with theoretical predictions [Phys. Rev. E 73, 056607 (2006)]. Within this band the wire medium slab is capable of transmitting images with $\lambda / 15$ resolution irrespectively of the shape and complexity of the source. Actual bandwidth of operation for particular near-field sources can be larger than the ultimate value but it strongly depends on the configuration of the source.

PACS numbers: 78.20.Ci, 42.70.Qs, 42.25.Fx, 73.20.Mf
\end{abstract}

An original concept of imaging with resolution smaller than the wavelength (sub-wavelength imaging) has been recently proposed in [1]. It has been demonstrated that a regular array of parallel conducting wires (see Fig. 团(a)) is capable of transporting images with sub-wavelength details from one planar interface to another. The principle of operation of this device (see [2]) is based on the idea of transforming the whole spatial spectrum of the sub-wavelength source to the propagating modes inside of a metamaterial formed by the array of wires, also called as the wire medium [3]. In such a way, the evanescent waves which carry sub-wavelength information and normally decay in free space can be transformed into the propagating modes inside of the wire medium and transported to significant distances.

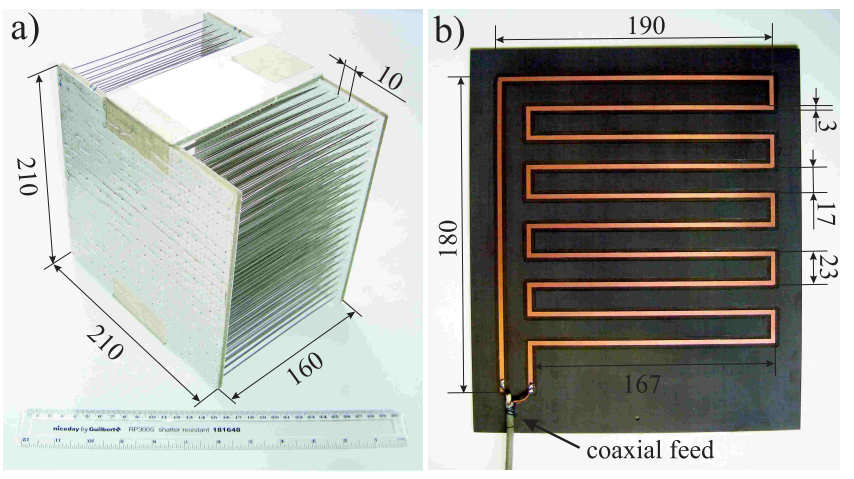

FIG. 1: (Color online) The geometries of the transmission device (a), an $21 \times 21$ array of wires with $1 \mathrm{~mm}$ radii, and the near-field source (b). All dimensions are in millimeters.

The initial experimental investigation of the subwavelength imaging capability of the wire medium slab has been performed recently in [1]. The antenna in the form of letter ' $\mathrm{P}$ ' was used as a sub-wavelength source. The clear images of the source were detected at the back interface of the transmission device and resolution of $\lambda / 15$ was demonstrated for $18 \%$ operation bandwidth. The extensive theoretical studies [4] based on analysis of transmission and reflection coefficient predicts that the sub-wavelength imaging should be observed for at least $4.5 \%$ bandwidth for any kind of the source. However, in practice, for certain sources the imaging can be observed within larger frequency bandwidths. The complexity of the near field produced by the source and the interaction between the source and the transmission device play crucial roles in determining the imaging performance of the whole system. At the frequencies outside of the theoretical minimum band of operation the strong reflections from the wire medium slab are expected in accordance to [4]. That is why the sensitivity of the source with respect to external fields becomes an issue. If the source is very complex and contains a lot of sub-wavelength details then its near field distribution can be easily deformed by harmful reflections from the interface of the transmission device and no proper sub-wavelength imaging can be observed at the frequencies outside of the theoretical minimum band of operation. However, if the source is simple and does not contain many sub-wavelength details then the source is immune from reflections and the image can be successfully transported to the back interface even at some frequencies outside of the minimum band of operation, as it was observed in [1], for example.

In order to investigate the imaging capability of the wire medium slab in details we have performed an experiment with the meander-line antenna printed on $2 \mathrm{~mm}$ thick slab of duroid with relative permittivity $\varepsilon=2.33$ (see Fig. 1(b) for other dimensions), which intentionally has much more complex near-field distribution as compared to the ' $\mathrm{P}$ ' antenna used in [1], see Fig. 2] The return loss $\left(S_{11}\right.$ parameter $)$ within the frequency band from $840 \mathrm{MHz}$ to $1060 \mathrm{MHz}$ for the meander-line antenna in the free space was compared with the return loss of the same antenna but placed close to the front interface of the wire medium slab, see Fig. 1(a). The results of the comparison are presented in Fig. [3] and clearly demonstrate that the wire medium slab does not affect the meanderline antenna at the frequency band from $915 \mathrm{MHz}$ to 955 $\mathrm{MHz}$, see the shaded area in Fig. 3 It means that within 915-955 MHz frequency range the meander-line antenna practically does not suffer from reflections from the wire 


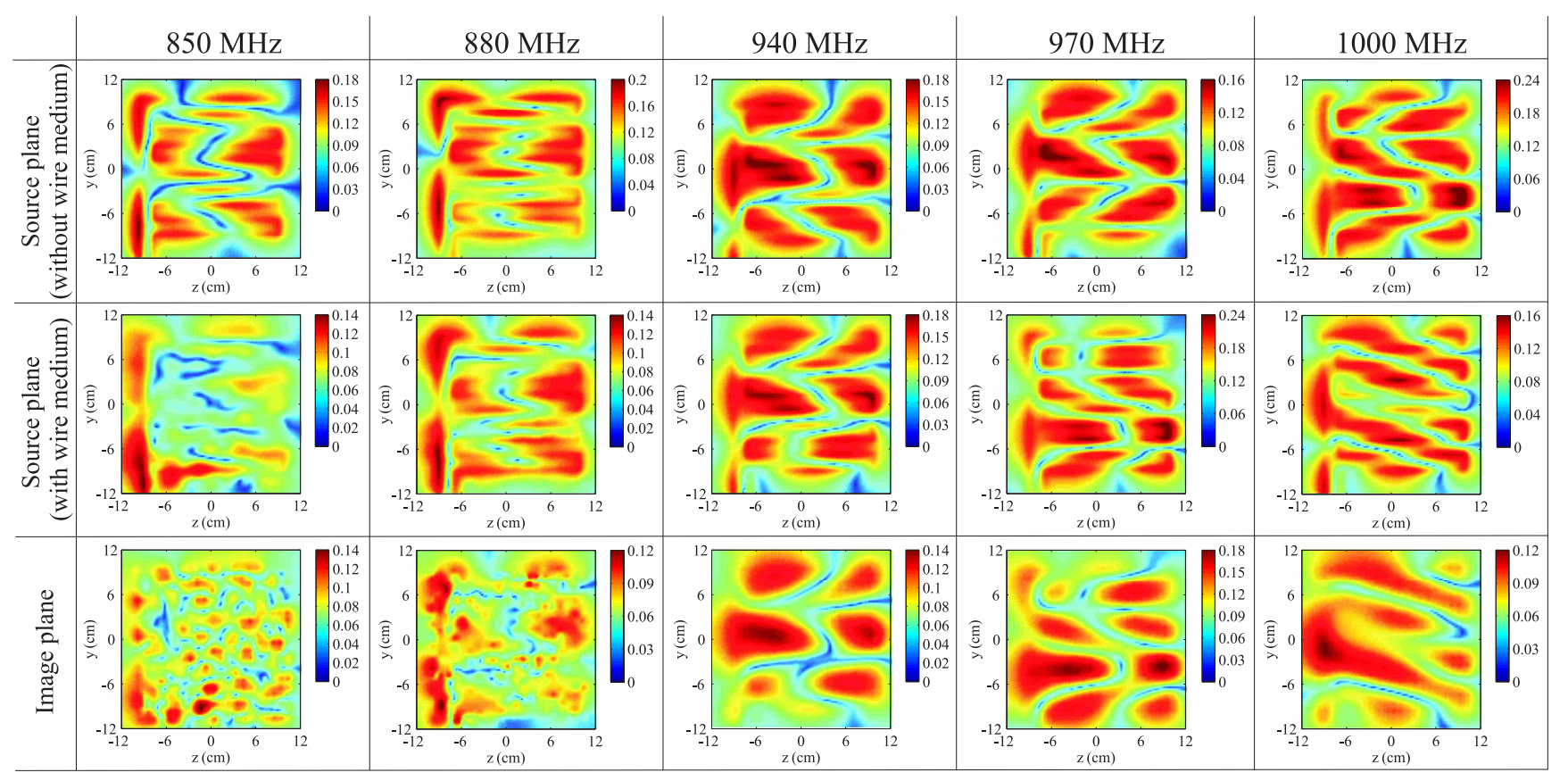

FIG. 2: (Color online) Results of the near field scan at $850 \mathrm{MHz}, 880 \mathrm{MHz}, 940 \mathrm{MHz}$ and $1 \mathrm{GHz}$ (in arbitrary units): the amplitude of the component of electric field normal to the interface at $2 \mathrm{~mm}$ distance from the meander antenna in the free space (source plane without wire medium), the same but when the antenna is placed at the front interface of the transmission device (source plane with wire medium) and at $2 \mathrm{~mm}$ distance from the back interface of the wire medium slab (image plane).

medium slab. The slab is practically transparent at these frequencies and this fact was predicted theoretically in [4] where unprecedentedly small values of reflection coefficient for all angles of incidence including evanescent waves have been demonstrated.

The near field scan has been performed for the frequencies within $840-1060 \mathrm{MHz}$ frequency band which is significantly wider than the band of $915-955 \mathrm{MHz}$ where perfect imaging is expected in order to verify general behavior of the imaging system. We used an automatic mechanical near-field scanning device and a $2 \mathrm{~mm}$ long monopole probe made from the central core of a coaxial cable with $2 \mathrm{~mm}$ diameter. The scan area was $24 \times 24$ $\mathrm{cm}^{2}$ with 75 steps in both directions. The probe was ori-

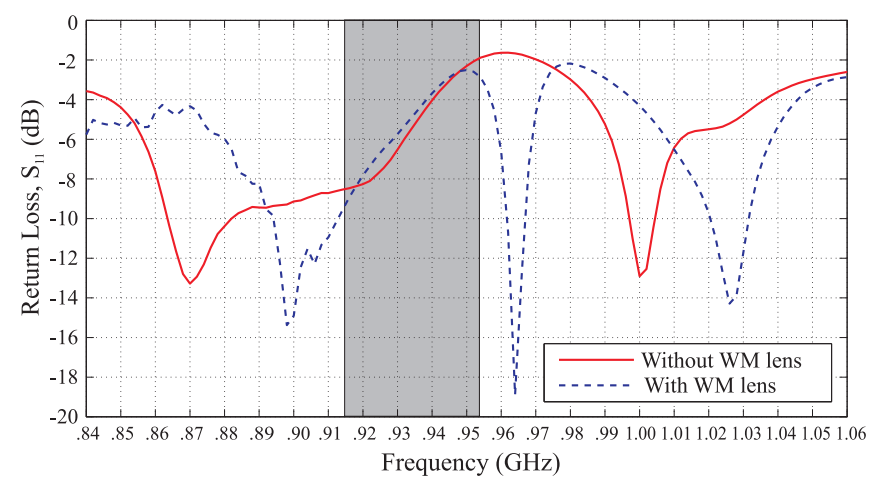

FIG. 3: (Color online) The return loss $\left(S_{11}\right.$ parameter) as function of frequency for the meander-line antenna in the free space and at the interface of the wire medium slab. ented normally with respect to the interfaces of both the meander antenna and the transmission device. So, it detected only the normal component of electrical field. The wire medium slab is capable of imaging only the electromagnetic waves with TM (transverse magnetic) polarization [1] and only the normal component of electric field is completely restored at the back interface. The other two components contain contribution of electromagnetic waves with $\mathrm{TE}$ (transverse electric) polarization, which are not transferred by the wire medium slab.

The slab of wire medium is a transmission device, not a usual lens. It transports electric field from its front interface to the back interface and does not involve any focusing effects. The electric field at $2 \mathrm{~mm}$ distance from the front interface of the meander-line antenna located in free space (without the wire medium) was scanned and regarded as the source field. After that the meanderline antenna was placed at the front interface of the wire medium slab and the field at $2 \mathrm{~mm}$ distance from the front interface of the antenna was scanned once gain. This allows us to detect the difference between the field created by antenna with and without the presence of wire medium. The image field was scanned at $2 \mathrm{~mm}$ distance away from the back interface of the slab in order to avoid touching of the probe and the transmission device. Results of the near-field scan at 23 frequencies from 840 $\mathrm{MHz}$ to $1060 \mathrm{MHz}$ with $10 \mathrm{MHz}$ step are presented in the multimedia file 5]. The same results, but only for $850 \mathrm{MHz}, 880 \mathrm{MHz}, 940 \mathrm{MHz}$ and $1 \mathrm{GHz}$ are shown in Fig. 2. At 910-960 MHz the fields at the source plane with 
and without presence of antenna are practically identical (see [5] or Fig. 2 for result at $940 \mathrm{MHz}$ ). This confirms that the wire medium slab practically does not introduce reflections at theses frequencies. At the same time the field in the image plane repeats the source field with accuracy about $2 \mathrm{~cm}$. This confirms that the resolution of the imaging device at this frequencies is about $\lambda / 15$.

At frequencies lower than $920 \mathrm{MHz}$ (up to $870 \mathrm{MHz}$ ) the fields in the source plane with and without wire medium slab remain practically identical. However, the image is distorted by sharp maxima (see [5] or Fig. 2 for result at $880 \mathrm{MHz}$ ). These maxima are caused by surface waves excited at the interfaces of the wire medium and were predicted theoretically in [4]. We can say that at these frequencies the transmission device maintains the capability of sub-wavelength imaging, but with reduced resolution. At frequencies lower than $870 \mathrm{MHz}$ the surface waves completely degrade the image and simultaneously provide strong reflections which make distribution in the source and image planes different.

At frequencies higher than $960 \mathrm{MHz}$ the imaging performance of the transmission device also degrades, but this happens because of other reasons. At $970 \mathrm{MHz}$ the distributions at the source plane with and without wire medium already become significantly different. It can be explained by strong reflections from the slab which change distribution of currents in the antenna. In this case reflections are much more prominent than those at lower frequencies and are caused by the fact that the slab does not fulfill the Fabry-Perot resonance condition anymore. Following the theoretical studies [4], at lower frequencies the reflection coefficient is large only for spatial harmonics with wave vectors close to the wave vector of the surface wave. That is why while the wave vector of the surface wave is large $(870-920 \mathrm{MHz})$ we observe only sharp maxima in the image plane and no significant changes between fields in the source plane with and without wire medium. As the wave vector of the surface wave decreases $(<870 \mathrm{MHz})$ the reflections experienced by the antenna from the wire medium increase and completely destroy the imaging. In the case of high frequencies $(>960 \mathrm{MHz})$ there are no surface waves, but the reflection increases and this increase happens simultaneously for all spatial harmonics. That is why we observe strong difference between fields in the source plane with and without wire medium at these frequencies. However, it is interesting to note that the distributions in the source and image planes of the transmission device remain practically identical (see [5] or Fig. 2 for result at $970 \mathrm{MHz}$ and $1 \mathrm{GHz}$ ). The difference is practically negligible at $960-1060 \mathrm{MHz}$. The resolution remains the same $(2 \mathrm{~cm}$, about $\lambda / 15)$ as at lower frequencies. Following the theoretical predictions [4] the resolution should slightly degrade with an increase of frequency, however, within the tested frequency range we were not able to detect any significant degradation of resolution.
Thus, we can conclude that the wire medium slab has good sub-wavelength imaging properties even at frequencies higher than the frequency of Fabry-Perot resonance, but the large level of reflections from the wire medium slab is an issue. If the sub-wavelength source is sensitive to external field (for example, the meander-line antenna whose current distribution changes in an external field), then the wire medium slab can not be used for its imaging. However, if the source field is not sensitive to external fields (for example, an array of small antennas fed by fixed current sources), then it remains unaffected by reflections from the transmission device and the wire medium slab can be successfully used for imaging of this source with very good sub-wavelength resolution. The antenna in the form of P-letter used in [1] is insensitive to reflections from the wire medium slab and that is why the sub-wavelength imaging with $\lambda / 15$ resolution was reported in [1] for the range from $920 \mathrm{MHz}$ to $1.1 \mathrm{GHz}$.

In conclusion, in this letter the minimum operation bandwidth of the wire medium slab as the subwavelength imaging device, theoretically predicted in [4], was confirmed experimentally. We would like to stress that the actual bandwidth of operation significantly depends on the complexity and sensitivity of the source to reflections from the wire medium slab. For sources which are not sensitive to external fields the sub-wavelength imaging can be performed within significantly wide frequency range. However, for an arbitrary source the imaging can be guaranteed only within the minimum bandwidth. We would like to remind [1, 4] that the wire medium slabs are able to transmit images to any long distances specified by particular application. The only restriction is that the length of the transmission device should be equal to an integer number of half-wavelengths in order to fulfill Fabry-Perot condition and eliminate unwanted reflections. The resolution of the wire medium slab is ultimately defined by its period. That is why in the microwave frequency range practically any fine subwavelength resolution can be obtained if the wire medium with the sufficiently small period can be manufactured.

[1] P. A. Belov, Y. Hao, and S. Sudhakaran, Phys. Rev. B 73, 033108 (2006).

[2] P. A. Belov, C. R. Simovski, and P. Ikonen, Phys. Rev. B. 71, 193105 (2005).

[3] P. Belov, R. Marques, S. Maslovski, I. Nefedov, M. Silverinha, C. Simovski, and S. Tretyakov, Phys. Rev. B 67, 113103 (2003).

[4] P. A. Belov and M. G. Silveirinha, Phys. Rev. E 73, 056607 (2006).

[5] measurment.mov (attached multimedia file). 